\title{
Rodolfo STAVENHAGEN
}

sociologue (El Colegio de Mexico)

(1963)

\section{"La réforme agraire et les classes sociales rurales au Mexique.”}

\author{
Un document produit en version numérique par Jean-Marie Tremblay, bénévole, \\ professeur de sociologie au Cégep de Chicoutimi \\ Courriel: jean-marie tremblay@uqac.ca \\ Site web pédagogique : http://www.uqac.ca/jmt-sociologue/ \\ Dans le cadre de: "Les classiques des sciences sociales" \\ Une bibliothèque numérique fondée et dirigée par Jean-Marie Tremblay, \\ professeur de sociologie au Cégep de Chicoutimi \\ Site web: http://classiques.uqac.ca/ \\ Une collection développée en collaboration avec la Bibliothèque \\ Paul-Émile-Boulet de l'Université du Québec à Chicoutimi \\ Site web: http://bibliotheque.uqac.ca/
}




\section{Politique d'utilisation de la bibliothèque des Classiques}

Toute reproduction et rediffusion de nos fichiers est interdite, même avec la mention de leur provenance, sans l'autorisation formelle, écrite, du fondateur des Classiques des sciences sociales, Jean-Marie Tremblay, sociologue.

Les fichiers des Classiques des sciences sociales ne peuvent sans autorisation formelle:

- être hébergés (en fichier ou page web, en totalité ou en partie) sur un serveur autre que celui des Classiques.

- servir de base de travail à un autre fichier modifié ensuite par tout autre moyen (couleur, police, mise en page, extraits, support, etc...),

Les fichiers (.html, .doc, .pdf., .rtf, .jpg, .gif) disponibles sur le site Les Classiques des sciences sociales sont la propriété des Classiques des sciences sociales, un organisme à but non lucratif composé exclusivement de bénévoles.

Ils sont disponibles pour une utilisation intellectuelle et personnelle et, en aucun cas, commerciale. Toute utilisation à des fins commerciales des fichiers sur ce site est strictement interdite et toute rediffusion est également strictement interdite.

L'accès à notre travail est libre et gratuit à tous les utilisateurs. C'est notre mission.

Jean-Marie Tremblay, sociologue

Fondateur et Président-directeur général, LES CLASSIQUES DES SCIENCES SOCIALES. 
Cette édition électronique a été réalisée par Jean-Marie Tremblay, bénévole, professeur de sociologie au Cégep de Chicoutimi à partir de :

\section{Rodolfo STAVENHAGEN}

[sociologue (El Colegio de Mexico)]

“La réforme agraire et les classes sociales rurales au Mexique.”

Un article publié dans la revue Cahiers internationaux de sociologie, vol, 34, janvier-juin 1963, pp. 151-164. Paris : Les Presses universitaires de France. Études critiques.

[Autorisation formelle accordée par l'auteur le 9 juillet 2003 de diffuser toutes ses publications dans Les Classiques des sciences sociales.]

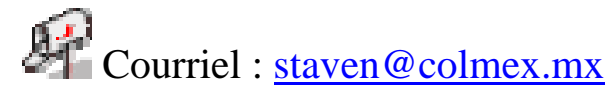

Polices de caractères utilisée :

Pour le texte: Times New Roman, 14 points.

Pour les citations : Times New Roman, 12 points.

Pour les notes de bas de page : Times New Roman, 12 points.

Édition électronique réalisée avec le traitement de textes Microsoft Word 2004 pour Macintosh.

Mise en page sur papier format : LETTRE (US letter), 8.5’’ x 11'’)

Édition numérique réalisée le 16 juin 2008 à Chicoutimi, Ville de Saguenay, province de Québec, Canada.

\section{Fait avec}

Macintosh 


\section{Rodolfo STAVENHAGEN}

sociologue (El Colegio de Mexico)

\section{"La réforme agraire et les classes sociales rurales au Mexique”}

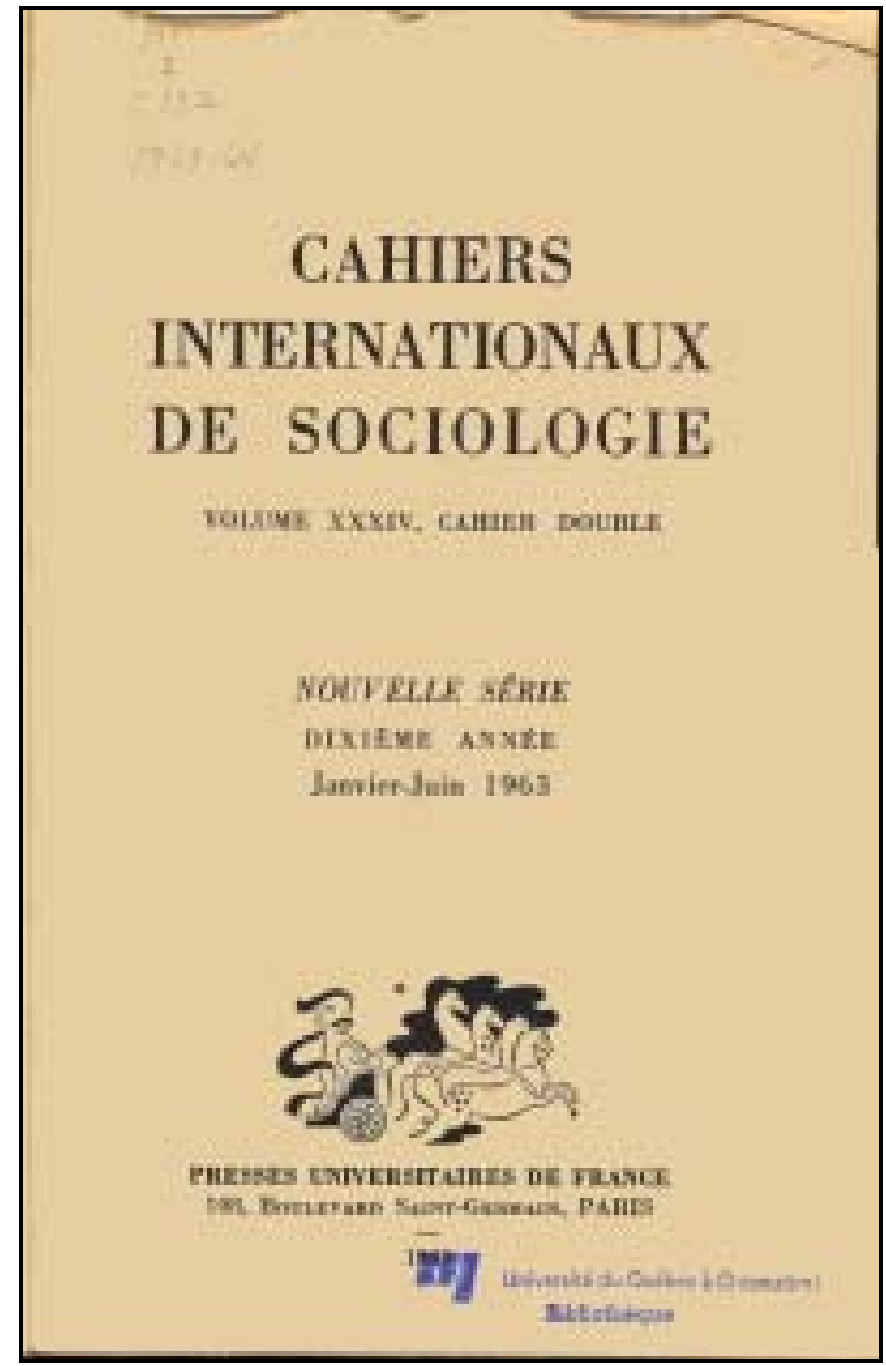

Un article publié dans la revue Cahiers internationaux de sociologie, vol, 34, janvier-juin 1963, pp. 151-164. Paris : Les Presses universitaires de France. Études critiques. 


\section{Table des matières}

Introduction historique

Aspects démographiques.

La propriété de la terre.

La Réforme agraire.

a) Les ejidatarios.

b) Les petits paysans parcellaires

c) Les sans-terre

d) La moyenne bourgeoisie paysanne

e) Les grands propriétaires fonciers

f) La couche paysanne intermédiaire

L'agriculture capitaliste et ses classes. 


\author{
Rodolfo Stavenhagen \\ sociologue (El Colegio de Mexico)
}

"La réforme agraire et les classes sociales rurales au Mexique”.

Un article publié dans la revue Cahiers internationaux de sociologie, vol, 34, janvier-juin 1963, pp. 151-164. Paris : Les Presses universitaires de France. Études critiques.

\title{
Introduction historique
}

$\underline{\text { Retour à la table des matières }}$

Le Mexique, avec le Pérou, est le seul pays de l'Amérique latine où une haute civilisation indienne lut détruite par la conquête espagnole. Quelques structures de l'agencement social qui existait alors survécurent longtemps à la Conquista et ne furent adaptées à la nouvelle structure sociale ou absorbées par celle-ci que lentement, après des décennies et parfois des siècles. Elles survivent encore en partie. D'autre part, il se développa une culture métisse qui présentait, et qui présente encore aujourd'hui, des traits particuliers aux civilisations espagnole et indienne ${ }^{1}$. Grâce à l'Indépendance politique, au début du XIXe siècle, une nouvelle classe s'installa au pouvoir, celle des criollos, c'est-à-dire celle des Blancs de souche espagnole nés dans le pays. L'incapacité à gouverner de cette classe dominante, réactionnaire et tournée vers l'étranger, apparaît dans le fait qu'elle ne put défendre le pays contre les agressions des États-Unis et de la France, permettant

1 De nombreuses observations faites par F. BOURRICAUD au Pérou (cf. Quelques caractères originaux d'une culture métisse en Amérique latino-indienne, in Cahiers Internationaux de Sociologie, vol. XVII, 1954), pourraient être transposées pour s'appliquer à la situation mexicaine. 
aux premiers d'annexer la moitié du territoire en 1848. Lors de la guerre de libération contre l'occupation française, de 1861 à 1867, se constitua comme classe une nouvelle bourgeoisie d'éléments métis qui composaient déjà à l'époque une grande partie de la population. L'organisation sociale de cette nouvelle période se caractérisa par la coalition de la bourgeoisie commerçante libérale avec l'aristocratie foncière ${ }^{2}$. La contradiction entre les conditions du développement de cette bourgeoisie urbaine dont la doctrine avait trouvé son expression dans la Constitution libérale de 1857, et les conditions mi-féodales, micapitalistes de la campagne, facilitèrent l'intervention des capitaux américains, français, anglais et allemands qui affluaient vers le pays. Bien que la situation économique de la population rurale n'apparut jamais clairement au premier plan des préoccupations, jusqu'à ce siècle, elle ne cessera de jouer un rôle important dans l'histoire du pays. Des soulèvements de paysans et d'Indiens boule. versèrent le pays avant comme après l'Indépendance. Mais pendant la longue guerre d'Indépendance qui dura -onze ans, les paysans armés jouèrent un rôle majeur, de même que dans la guerre civile qui opposa d'abord le gouvernement libéral au parti conservateur clérical, et qui se transforma plus tard en guerre de libération contre l'intervention française et l'Empire de Maximilien alors établi. La participation de la paysannerie au combat national peut être considérée comme l'expression des profondes contradictions propres aux situations agraires des différentes périodes. C'est ce que reconnurent souvent les dirigeants des mouvements politiques et révolutionnaires, bien qu'ils n'aient jamais appartenu à la paysannerie et qu'ils aient représenté le plus souvent les intérêts de la nouvelle classe dominante. Pendant la guerre d'Indépendance (1810-1821), comme pendant la guerre dite de la Reforma (18581860), se manifestèrent les débuts d'une solution radicale des problèmes agraires. Cependant, si les solutions qu'on adopta successivement ont transformé les conditions agraires, elles n'ont jamais favorisé

2 Voir l'article de Noël SALOMON, Féodalité et capitalisme au Mexique de 1856 à 1910, in La Pensée, nouvelle série, no 42-43, Paris, 1952. 
qu'une nouvelle classe montante sans améliorer la condition des paysans.

Cette situation s'aggrava pendant les trente dernières années du XIXe siècle, et aboutit en 1910 à une explosion. Cette fois, les paysans menèrent leur propre lutte. Les propriétés foncières et les conditions semi-féodales qui empêchaient le développement capitaliste de l'agriculture furent liquidées définitivement. Dans la nouvelle structure du pays, et pour la première fois, la paysannerie joua un rôle politique et fut acceptée politiquement comme un élément constructif du développement économique.

\section{Aspects démographiques.}

$\underline{\text { Retour à la table des matières }}$

La paysannerie représente encore aujourd'hui la majorité de la population mexicaine. Selon les statistiques officielles (1958), 55\% de la population vivait en milieu rural. Cependant, la proportion de la population rurale relativement à la population totale décroît : elle était de $70 \%$ en 1930 , de $65 \%$ en 1940 et de $57 \%$ en 1950 ' ${ }^{3}$ Si nous considérons la population active, nous constatons qu'en 1955, 58\% d'entre elle se consacrait à l'agriculture.

La moitié de la population du Mexique vit dans la région centrale du pays qui ne représente qu'un sixième du territoire, et dans les zones situées à plus de 1500 mètres d'altitude. Sur près de 100000 agglo-

3 Néanmoins, on devrait augmenter ces chiffres officiels de 8\% pour des raisons sociologiques. Dans les statistiques officielles, sont considérées comme rurales les localités de 2500 habitants et moins. Mais on peut y ajouter les localités de 2500 à 5000 habitants, car elles présentent toutes les caractéristiques de la vie rurale. C'est alors $63 \%$ de la population totale qui compterait comme rurale. 
mérations, 65000 ont moins de 100 habitants, et 25000 ont entre 100 et 500 habitants.

Les transformations sociales au cours des dernières décennies ont aussi modifié la structure démographique de la population paysanne. L'exode rural continu pendant les dernières années est l'un des facteurs importants de cette transformation. Entre 1930 et 1958, la population rurale s'est accrue de $62 \%$, tandis que celle des villes augmentait de $160 \%$. Une autre tendance de cette population paysanne est son immigration orientée de plus en plus vers le Nord du Mexique, resté jusqu'alors peu peuplé. Il y a plusieurs raisons à cette émigration : la dégradation des conditions de vie de la paysannerie ; les meilleures conditions de travail offertes dans les villes industrielles et dans quelques régions nouvellement irriguées du Nord; enfin, l'émigration temporaire annuelle de plus d'un million de journaliers agricoles, braceros, vers les États-Unis.

Grâce aux mesures sociales prises par le gouvernement et à l'amélioration des conditions de l'hygiène, la mortalité a baissé au cours des dernières années, et la natalité a augmenté. Le problème dû à la surpopulation qui s'ensuit ne peut pas être résolu dans le cadre de la situation actuelle, comme c'est le cas d'ailleurs dans la plupart des pays sous-développés du monde capitaliste.

\section{La propriété de la terre.}

Retour à la table des matières

Avant la Conquête espagnole, les peuples nomades du Nord du pays vivaient encore sous le régime de la communauté primitive. Le concept de propriété ne s'était développé que parmi les peuples sédentaires et agricoles du Mexique méridional. Les civilisations supérieures, et surtout celle des Aztèques, connaissaient différentes formes de propriété. La plus répandue correspondait aux propriétés collectives des clans (Calpulli) dans lesquelles chaque membre avait droit à un 
champ aussi longtemps qu'il le cultivait régulièrement. Cependant, une sorte de propriété privée de la terre existait chez les Aztèques lorsque la Conquête détruisit brutalement la civilisation indienne. Les Espagnols mirent en place un système qui, d'un côté, maintenait la propriété collective des villages indiens, mais qui, d'un autre, créait les Encomiendas, régime par lequel les villages indiens d'un district déterminé devaient payer un tribut à un seigneur féodal espagnol. Par ailleurs, se constituait la propriété foncière privée des Espagnols, qui se conforma à des modalités imposées par la Couronne ; et à partir du XVIIe siècle se développaient les grands domaines, les haciendas. Les limitations imposées à la propriété foncière soulevaient le mécontentement des criollos et de certaines autres couches sociales. Ce n'est que pendant la période de l'Indépendance et après la guerre de la $R e$ forma que la propriété foncière privée fut libérée totalement de toutes ses entraves juridiques. L'Église, qui resta jusqu'au milieu du siècle le plus grand propriétaire foncier et la plus puissante institution financière du pays, fut expropriée, de même que les villages indiens, dont les terres communes se transformèrent, au nom du libéralisme économique, en propriété privée.

Pendant la deuxième moitié du siècle, la propriété foncière ne cessa de se concentrer, et la hacienda devint l'expression typique de la structure agraire. La hacienda se caractérisait ainsi : les peones, les paysans sans terre, étaient réduits au servage. Les propriétaires ou leurs intermédiaires exerçaient un pouvoir brutal sur leurs misérables ouvriers agricoles, qui étaient liés à la terre par l'esclavage pour dettes et par d'autres chaînes. Les haciendas étaient des cellules économiques à peu près autonomes qui vivaient dans une relative autarcie, plus ou moins à l'écart du faible marché national. En 1910, dernière année de la dictature de Porfirio Diaz, $1 \%$ de la population possédait 97\% de la superficie du pays. Une partie de ces latifundia appartenait à des sociétés anonymes ou à des propriétaires privés étrangers. Quelques-unes des haciendas s'étendaient sur plus de 300000 ha. Deux pour cent seulement du territoire se trouvait aux mains des petits paysans indépendants. Inversement, 96\% de la population rurale pos- 
sédait 1\% seulement du territoire. Sur les 70000 agglomérations qui existaient alors au Mexique, 55000 (soit la moitié de la population) se trouvaient à l'intérieur des haciendas. La minorité dominante accordait toute son aide aux grands intérêts étrangers pour exploiter les richesses naturelles du pays et ses ressources humaines. Elle vivait ellemême dans un luxe qui faisait ressortir par contraste la misère des masses paysannes.

Cette misère est à l'origine de la Révolution de 1910 qui dura plus de dix ans. La Révolution se caractérisa dès le début par une revendication agraire, déjà formulée par ses idéologues. La lutte du chef des paysans, Émiliano Zapata, est aujourd'hui légendaire. Il donna à la Révolution son programme spécifiquement agraire et fut le premier à distribuer la terre aux peones. D'autre part, dans le Nord, les campagnes de Francisco ( $P$ Pancho ») Villa entraînèrent la moitié du pays dans la Révolution et provoquèrent l'intervention militaire des ÉtatsUnis en 1917. Ainsi, les différents gouvernements révolutionnaires furent amenés à promulguer des lois qui devaient résoudre une fois pour toutes le brûlant problème agraire, lois qui trouvèrent leur expression définitive dans la nouvelle Constitution de 1917.

\section{La Réforme agraire.}

$\underline{\text { Retour à la table des matières }}$

On entend souvent dire que la Révolution mexicaine avait trop de programmes et pas assez d'idéologie. Malgré les tendances divergentes des différents groupes et dirigeants qui se disputèrent le pouvoir, la solution du problème agraire était l'idée fondamentale de la Révolution, celle qui lui a donné, historiquement, son unité et son but. Dès les premières années de la révolte, et avant même que le programme agraire de la Révolution ne se soit fixé, la terre était déjà répartie par les groupes rebelles, en tous les points de la nation. 
La Réforme agraire qui fut réalisée légalement dès 1915, avait pour buts de donner la terre aux paysans qui n'en avaient pas, en partageant les grandes haciendas, et d'éviter une nouvelle concentration foncière qui remettrait la terre aux mains d'une minorité. Il ne fut pas question de socialisation au de collectivisation. Il ne s'agissait que de donner au petit paysan une propriété et de la protéger. Cependant, cette tentative n'aboutit pas. Elle se heurta au niveau culturel et matériel très bas des paysans, en même temps qu'elle freina le développement technique et économique de l'agriculture. La Réforme prévoyait deux formes légales de la propriété foncière.

La première correspond à la propriété privée classique, dont la fermes de l'Europe de l'Ouest et du Contre devaient être le modèle. La seconde, qu'on appelle Ejido, était une nouvelle forme de propriété foncière, dont on peut trouver les origines à la fois dans les calpulli de l'ancienne communauté des clans aztèques, et plus encore dans les vaines pâtures des villages de l'Espagne médiévale. L'ejido se compose, d'une part, de terres en principe réservées à la culture individuelle, et d'autre part, de pâturages et de bois communs. Ces ejidos sont placés par l'État sous l'administration de communautés composées d'individus dépourvus de terre. Tout homme valide de la commune, remplissant certaines conditions prévues par la loi, se consacrant régulièrement à l'agriculture, et ne disposant d'aucun autre revenu, reçoit le droit de cultiver une partie des terres de l'ejido. Ce droit n'est, en aucune façon, un droit de propriété privée. La terre appartient à la nation et la communauté peut la cultiver. Le paysan conserve son droit aussi longtemps qu'il habite dans le village et aussi longtemps qu'il cultive régulièrement sa parcelle. Ni les parcelles, ni l'ejido entier ne peuvent être vendus, hypothéqués, prêtés, loués ou échangés. En dehors des terres cultivables attribuées aux paysans, les pâturages, les bois et l'eau sont destinés à servir au village en commun. Il est prévu que la terre des ejidos doit provenir de l'expropriation des haciendas proches des villages. 
Telle était la base légale des ejidos. Le but dé cette institution était essentiellement de permettre aux ouvriers agricoles travaillant comme péons dans les grandes propriétés, de compléter leur misérable salaire. Mais il apparut bientôt que la logique de la Réforme agraire conduisait à la suppression du système des haciendas, Le mot d'ordre de la révolution des paysans avait été l'appel de Zapata, «Tierra y Libertad », et pas un seul des peones libérés n'était prêt à retourner sur la terre du seigneur. L'ejido fut alors conçu comme une étape vers la formation d'une petite propriété paysanne, en attendant que l'ouvrier agricole, illettré, traditionaliste et sans connaissances techniques, se transforme en un paysan moderne. Bien des années s'écoulèrent avant que l'ejido, ne fût reconnu comme forme définitive do la propriété foncière. Pendant les années qui suivirent la Révolution, la distribution des terres progressa relativement lentement. Elle atteignit son point culminant sous la gouvernement du président Lázaro Cárdenas (1934-1940). Depuis 1940, le partage et la Réforme agraire en général ont été très ralentis, Cependant, il semble que pondant les deux dernières années, après le récent changement de gouvernement, on leur ait insufflé à nouveau un peu de vie.

Quelques chiffres montreront la situation actuelle en, ce qui concerne la répartition de la terre au Mexique. Selon les dernières statistiques, 40 millions d'hectares appartenaient à la fin de 1960 aux ejidos, soit à pou près $27 \%$ du territoire. Ces terres sont réparties entre plus de 18000 ejidos, Sur lesquels deux millions de paysans environ possèdent un droit d'usufruit. Ces paysans, les ejidatarios, représentent environ $18 \%$ de la population active totale et environ $30 \%$ de la population active agricole. Malgré l'étendue des terres placées sous le régime de l'ejido, chaque ejidatario dispose en moyenne de 6,8 ha de terre cultivable. Ce seul chiffre montre déjà l'insuffisance du partage des terres comme solution au problème agraire, surtout si l'on considère le niveau technique et les rendements relativement bas de l'agriculture mexicaine. Soixante-treize pour cent du territoire se trouve encore être l'objet d'une appropriation privée. Deux pour cent de ces propriétés privées appartiennent à plus d'un million de paysans, et leur 
superficie est en moyenne de 1,5 ha. Quant au reste, c'est-à-dire la plus grande partie du pays, il est réparti entre quelques milliers de propriétés ayant 300 ha de superficie en moyenne. Par contre, plus de deux millions d'ouvriers agricoles, complètement dépourvus de terre, travaillent à la campagne. Ce sont les journaliers et les braceros qui vont souvent aux Etats-Unis chercher du travail.

Ces chiffres ne suffisent cependant pas à donner une idée des conditions réelles qui prévalent dans les campagnes. La Réforme agraire du Mexique ne s'est pas limitée à une répartition des terres. Le morcellement en petites parcelles individuelles n'est qu'une solution partielle au problème agraire, qui a satisfait les premières aspirations du mouvement paysan. Les anciennes haciendas représentaient, cependant, des unités économiques de production qui, dans certains cas, s'étaient transformées en grandes entreprises capitalistes, spécialement en ce qui concerne les propriétés d'élevage au nord et quelques plantations de riz, de sucre et de bananes au sud. Ces entreprises furent liquidées par la répartition des terres. C'est seulement plusieurs années après la Révolution, que la production agricole retrouva le niveau antérieur. Plusieurs théoriciens de la Réforme agraire avaient déjà indiqué que la répartition aveugle et indistincte des terres était économiquement dangereuse. Ils proposaient la collectivisation. Ce n'était pas les haciendas en tant qu'unités qu'il fallait détruire, mais leur organisation. En plusieurs points du territoire mexicain, les paysans avaient pris d'eux-mêmes de semblables initiatives. Bien que la collectivisation n'entrât jamais au programme gouvernemental, quelques ejidos collectifs furent créés, véritables coopératives de production, spécialement dans les régions de cultures commerciales.

Comparés à la répartition des terres, pourtant si peu achevée, les autres buts de la Réforme agraire sont encore plus loin d'être atteints. On peut qualifier la situation économique de l'agriculture mexicaine de favorable si on la compare à celle d'il y a 50 ou 30 ans, ou à celle d'autres pays sous-développés. Cependant, il est évident que le développement de l'agriculture ne suit pas celui des autres secteurs de 
l'économie ${ }^{4}$. Le crédit agricole, les zones d'irrigation, la mécanisation du travail agricole, l'amélioration du niveau technique d'exploitation, les prix de vente garantis des récoltes, l'organisation du marché agricole, etc., tout cela fut mis en train à différentes époques, et seulement partiellement réalisé.

Les secteurs de l'agriculture qui sont en progrès se développent d'abord en faveur d'une nouvelle et puissante bourgeoisie issue de la Révolution, mais aux dépens de l'indépendance économique et du standard de vie de la majorité de la population. Les résultats acquis se répartissent irrégulièrement entre diverses fractions de la population rurale. En 1950, le revenu annuel moyen d'un propriétaire de moins de 5 ha était d'environ 40 dollars, celui d'un ejidatario d'environ 115 dollars, et celui d'un propriétaire de plus de 5 ha autour de 800 dollars. Ces catégories représentaient respectivement $36 \%$, $54 \%$ et $10 \%$ des exploitations agricoles.

Dans cette deuxième partie de notre étude, nous essaierons de caractériser les classes sociales à la campagne et de déterminer leurs relations respectives. Si l'étude des strates et de la stratification représente surtout une démarche descriptive, celle des classes exige un procédé analytique et structurel et une méthode dialectique. À l'opposé de la strate, comme catégorie statistique, le concept de "classe » est une catégorie analytique. Les classes «sont des groupements d'intérêts issus de certaines conditions structurelles, qui participent comme telles dans les conflits sociaux et contribuent aux transformations des structures sociales ${ }^{5}$. Les classes ne peuvent exister que dans des

4 Le revenu de l'agriculture représentait en 1950 14\% du revenu national, bien que $60 \%$ à peu près de la population active se trouve dans ce secteur. Sur la base de $1939=100$, le revenu dans l'agriculture en 1950 se trouvait à l'indice 183 ; mais dans l'industrie (excepté construction et électricité) à 228, et dans le commerce et les services privés à 251. Cf. Comisión mixta, El desarrollo económino de México y su capacidad para absorber capital del exterior, Nacional Financiera, S.A., Mexico, 1953.

5 Cf. Ralf DAHRENDORF, Soziale Klassen und Klassenkonflikt in der industriellen Gesellschalt, Stuttgart, 1957, p. ix. 
systèmes de classes. Elles représentent des forces sociales et historiques qui, au cours de leur développement, entrent en opposition les unes avec les autres, dans le cadre spécifique des structures socioéconomiques. Les grands groupes humains qui vivent à la campagne au Mexique ont une véritable existence (groupements de fait) qui est conditionnée par leur position dans la production et par leurs rapports avec les moyens de production (la terre, surtout), dans le cadre de structures économiques régionales bien déterminées.

Les nouvelles strates sociales. - Avant 1910, il n'y avait dans les campagnes mexicaines que deux classes principales qui s'opposaient : les peones, journaliers agricoles, qui ne possédaient pas de terres et les grands propriétaires fonciers. Leurs rapports réciproques étaient nets et la lutte qui les opposa fut sans limites, violente et révolutionnaire. Aujourd'hui les conditions agraires se présentent différemment. Plusieurs classes et strates sociales se sont développées. Leurs relations réciproques et celles qu'elles entretiennent avec les autres classes de la société mexicaine est complexe, et les conflits de classes s'expriment de plusieurs façons.

a) Les ejidatarios. - Presque deux millions de paysans travaillent dans les ejidos. Véritable produit de la Révolution et de la Réforme agraire ; ils sont liés par une législation et des institutions spéciales (y compris une "Banque agricole nationale») au secteur officiel de l'économie, Cette fraction de la paysannerie peut être considérée comme progressiste et révolutionnaire, dans la mesure où les ejidos sont nés de ta lutte révolutionnaire et où les membres actuels des ejidos où leurs pères se sont organisés politiquement pour s'emparer de la terre. Les ejidos, en effet, ne sont pas créés de plein droit par le gouvernement, ils ne sont installés que lorsqu'une collectivité paysanne dénonce l'existence d'un grand domaine qui n'a pas encore été partagé et demande son partage. Cela ne veut pas dire que toutes les requêtes soient satisfaites. Il y a des milliers de communes qui ont fait leur requête depuis des années et qui attendent encore que le gouvernement leur donne satisfaction, L'ejido est donc une communauté 
économique et politique organisée. La caractéristique principale est le contrôle collectif de la terre, sous les normes établies par la loi. Comme la terre occupée par ces paysans n'est pas une propriété privée, elle a le plus grand intérêt à voir la réforme agraire se poursuivre et se développer, spécialement sous ses aspects techniques et économiques.

Cependant, différentes forces s'opposent conjointement au développement de cette catégorie sociale et politique, La majorité des paysans des ejidos cultivent individuellement leur parcelle. Du point de vue économique, ce sont de petits paysans, qui n'ont ni la possibilité d'accumuler un capital, ni d'investir sur une terre qu'ils cultivent mais qui ne leur appartient pas. Ils ne peuvent pas être légalement dépossédés, mais ils voient leur influence diminuer lentement parce que leur participation au revenu national décroît. La nouvelle bourgeoisie bureaucratique, qui grandit à l'ombre des gouvernements « révolutionnaires ", n'hésite pas non plus, quand c'est dans son intérêt, à détruire l'organisation de certains ejidoa, avec la complicité d'autorités locales corrompues.

Deux sortes de critiques sont généralement faites au système de l'ejido ; l'une n'y voit qu'une survivance d'une conception moyenâgeuse de l'organisation agricole, gênante pour le développement capitaliste ; l'autre, consciente du fait que le système des ejidos ne peut subsister sans un soutien dynamique du gouvernement, tire argument de son actuelle insuffisance économique pour préconiser une collectivisation réelle de l'agriculture.

b) Les petits paysans parcellaires. - Cette seconde fraction de la paysannerie se compose de propriétaires de terre ne dépassant pas 5 ha, L'étendue de leur propriété ne permet pas la mécanisation de l'exploitation et les revenus qu'ils en tirent leur interdisent toute capitalisation, Vingt et un pour cent de la population active dans l'agriculture, soit en tout plus d'un million, se situent dans cette catégorie. Ils ne possèdent ensemble que $7 \%$ de la surface cultivable et gagnent moins de $2 \%$ du revenu du secteur privé agricole. Ils doivent leur minuscule 
propriété à la Révolution, mais n'en ont tiré que peu d'avantages. Ils se trouvent à l'échelon le plus bas du développement économique. Ils sont exposés aux aléas climatiques et géographiques, et ne disposent que d'une technique primitive : le bâton à fouiller, la houe et exceptionnellement l'araire. Tout changement de l'équilibre écologique aggrave leur situation. Ils se consacrent en général à la culture du maïs, des haricots et des courgettes, le même complexe alimentaire qui caractérisait déjà l'agriculture indienne de l'Amérique centrale avant la Conquête. Leur économie est une économie naturelle de subsistance, relativement autarcique. Une faible proportion de ce que chacun produit prend le chemin du marché national. Et pourtant, ils sont tous ensemble les principaux fournisseurs des marchés régionaux et locaux, car ils doivent continuellement vendre quelque chose pour subvenir aux besoins quotidiens les plus élémentaires.

Plus encore que leur autarcie et leur faible niveau de vie, ce sont leurs relations avec le marché qui caractérisent cette paysannerie. L'étude sociologique des marchés locaux -particulièrement dans les zones habitées par les Indiens - a été entreprise sporadiquement au Mexique avec des résultats prometteurs. Au marché local, les petits paysans parcellaires sont à la fois vendeurs et acheteurs, mais la faiblesse économique de chacun ne leur permet pas d'agir individuellement sur le niveau des prix. Ces marchés locaux se situent à l'extrémité la plus éloignée d'un système commercial dont l'autre pôle est représenté par le marché national, et au sein duquel se maintient une foule d'intermédiaires vivant au dépens du petit paysan. D'autre part, l'économie monétaire à laquelle participe partiellement celui-ci, renforce certains aspects de la structure sociale traditionnelle, en contribuant à l'économie de prestige, au maintien de certains types de relations matrimoniales, au compérage, etc., qui caractérisent la petite communauté folk, laquelle constitue le monde social du petit paysan.

La main-d'œuvre constituée par les membres de la famille de ces petits paysans suffit à la culture du champ. Cette paysannerie parcellaire est trop faible, sa production et sa productivité trop basses, pour 
être prise en considération par les grandes banques et les institutions de crédit gouvernementales. Elle est donc livrée aux spéculateurs, aux intermédiaires et aux usuriers locaux. Son économie présente un déficit chronique. C'est dans cette paysannerie que le sous-emploi se manifeste le plus, car la capacité de travail du petit paysan et de sa famille ne peut pas être utilisée entièrement dans le cadre restreint de sa minuscule propriété. Certains de ces paysans se placent comme métayers ou comme journaliers dans des entreprises agricoles importantes. Ils émigrent aussi temporairement dans les villes et dans les plantations de cultures commerciales. Beaucoup d'entre eux sont en outre artisans ou fabricants dans les petites entreprises familiales où ils produisent des articles de bois, de poterie, de tissage, de vannerie, etc., pour le marché national. Les relations sociales de cette paysannerie reposent sur les liens de parenté, du compérage (compadrazgo) et du voisinage ; mais sur le plan économique chaque petit paysan est abandonné à lui-même. Il est donc la proie la plus facile du petit capitalisme local.

Plus encore que les ejidatarios, cette couche souffre du développement capitaliste moderne. Elle est liée à la terre par la propriété et d'autant plus fermement que cette propriété a été conquise récemment par un dur combat. Mais le gouvernement a envers elle moins de devoirs qu'envers les ejidatarios. Elle reste en retard dans le développement de sa technique et de sa capitalisation, retard dû au fait de la limitation des petites surfaces qui sont la base de son existence. Elle a le même intérêt que les ejidatarios au développement de la Réforme agraire sur le plan technique et économique.

c) Les sans-terre. "La troisième catégorie sociale dont nous devons parler est composée de deux millions de paysans qui ne possèdent pas de terre. Comme ils représentent plus de $40 \%$ de la population active dans l'agriculture, ils révèlent que la Réforme agraire est 
encore loin d'avoir atteint ses objectifs ${ }^{6}$. Cette catégorie comprend elle-même trois fractions: les aides familiaux qui travaillent sur la propriété du chef de famille, les métayers et, surtout, les journaliers agricoles. Si les aides familiaux, qui représentent $18 \%$ de la population active de l'agriculture, paraissent ne pouvoir être considérés comme sans-terre, puisqu'ils sont liés par la parenté à celui qui possède la terre sur laquelle ils travaillent, ils le sont en réalité, car ces propriétés sont minuscules; ils sont les victimes du sous-emploi et du manque de terres disponibles. Les métayers, qui ne représentent que $4 \%$ de la population active dans l'agriculture, sont liés à l'économie agricole la plus arriérée du pays, d'un caractère quasi féodal. Les deux fractions que nous venons de mentionner se trouvent imbriquées dans un type d'économie que nous avons décrit à propos des petits paysans parcellaires. C'est dans le cadre d'une agriculture de subsistance qu'elles survivent, dans les régions les plus arriérées du pays.

C'est plutôt la fraction des journaliers agricoles, soit un million de paysans environ, qui retiendra notre attention. Bien qu'il s'agisse d'individus qui ont légalement droit à une terre, ils n'ont encore rien reçu de la Réforme agraire ni des régimes issus de la Révolution. Leurs revenus sont instables et souvent au niveau de subsistance le plus bas. Cette couche des sans-terre se compose en grande partie de paysans originaires du centre du pays, où la question agraire est brûlante, qui ont émigré vers la capitale ou vers les régions frontalières du Nord. D'un côté, ces paysans ont des tendances révolutionnaires qui se sont manifestées à plusieurs reprises, au cours des dernières années par l'occupation violente de domaines non partagés. D'autre part, ils sont relativement mal organisés, et ils sont affectés de diverses façons par le développement économique du pays. Une partie de cette paysannerie forme un prolétariat agricole dans les grandes exploitations des districts irrigués du Nord. Une autre reste à l'état de semi-prolétariat fixé tantôt dans les campagnes, tantôt dans les villes. Plusieurs mil-

6 Ces chiffres se rapportent à 1950, car les résultats du recensement de 1960 ne sont pas encore à notre disposition. 
liers d'entre eux travaillent comme journaliers dans les plantations du Texas, de la Californie et d'autres provinces des États-Unis, voisines du Mexique, mais ils restent régulièrement domiciliés dans les villes frontalières du Mexique. D'autres, en revanche, ceux qui sont appelés braceros, et qui sont aussi quelques centaines de mille, travaillent et résident une partie de l'année aux États-Unis. Beaucoup d'entre eux émigrent définitivement car ils ne peuvent plus gagner leur vie dans leur propre pays. D'autres encore, au sud et au sud-est, dans les parties les plus sous-développées du Mexique, et spécialement ceux qui appartiennent aux communautés indiennes, travaillent comme peones dans les grandes plantations, dans des conditions qui se rapprochent de celles des années d'avant la Révolution. L'existence et l'impatience croissante de ces sans-terre posent, pour un régime qui se veut révolutionnaire, un grave problème.

La bourgeoisie a besoin, d'une part, d'une main-d'œuvre libre qui ne soit pas liée à la terre ; mais d'autre part, ses positions restent menacées tant que n'est pas résolu le problème agraire. Si l'émigration annuelle de milliers d'ouvriers - 7\% de la population agricole active représente une perte de main-d'oeuvre nécessaire au développement du pays, d'un autre côté, elle atténue les risques politiques et économiques du chômage dans les campagnes, et elle est qualifiée par les économistes officiels de " soupape de sécurité ». Ce phénomène accentue la dépendance de l'économie mexicaine à l'égard de celle des États-Unis et expose aussi le bien-être d'une partie importante de la population mexicaine aux effets des crises économiques de ce pays.

d) La moyenne bourgeoisie paysanne. - La couche des paysans moyens détient des propriétés de 25 à 200 ha. Ces limites correspondent à des critères précis. Il a été calculé qu'à partir de 25 ha seulement l'emploi de l'énergie mécanique devient rentable. Il s'ensuit que ces propriétés, qui ont, en moyenne, quarante fois plus de terre cultivable que celles des petits paysans parcellaires, rapportent à leurs propriétaires un revenu moyen plus de 300 fois plus élevé que celui des petits paysans. En ce qui concerne la limite supérieure de 200 ha, ce 
chiffre représente l'extension maxima, permise par la loi, de la propriété privée agricole sur un territoire non irrigué.

Cette couche sociale provient de l'ancienne classe dominante des grands propriétaires fonciers qui ont perdu leurs latifundia lors de la Réforme agraire et qui ont le droit d'occuper encore, selon la loi, une fraction limitée de l'ancienne hacienda. En général, cette couche a perdu par la Révolution ses prérogatives et sa puissance d'autrefois. Elle n'apporte que rarement les investissements exigés par le développement capitaliste de l'agriculture. Cela est dû autant à son origine qu'aux limitations de la loi visant à prévenir le développement de la grande propriété. Les capitaux de cette moyenne bourgeoisie paysanne sont retirés de l'agriculture et placés dans d'autres branches de l'économie : commerce, spéculation foncière dans les villes de province, etc., ou bien, thésaurisée. Naturellement, des ouvriers agricoles travaillent sur ces propriétés. Des parcelles de terre sont affermées ou données en métayage à des petits paysans. Cependant, et c'est là une des caractéristiques essentielles de cette couche, le commerce et la spéculation représentent une part importante de ses activités, parfois même son activité principale. En outre, Il faudrait inclure dans cette catégorie sociale la plupart des gens qui ne se consacrent qu'au commerce dans les districts ruraux : commerçants, intermédiaires et usuriers qui s'enrichissent aux dépens du reste de la population paysanne.

Ils prêtent de l'argent à un taux usuraire et ils financent les récoltes. Parfois ces propriétaires commerçants se situent en haut de l'échelle nationale des revenus. Cette moyenne bourgeoisie paysanne, dans les conditions actuelles, représente une classe parasitaire qui, d'une part, exploite la population agricole et empêche son progrès et son émancipation, et qui, d'autre part, n'a aucun intérêt au développement d'un capitalisme progressiste dans l'agriculture.

e) Les grands propriétaires fonciers. - La couche des grands propriétaires fonciers, véritables capitalistes de l'agriculture, se compose an partie des propriétaires d'haciendas non encore partagées et aussi et 
surtout d'une nouvelle bourgeoisie issue de la Révolution, et qui, pendant les vingt dernières années s'est souvent dressée contre cette Révolution. Bien qu'en majeure partie produit de la Révolution et liée par là même aux différents cercles gouvernementaux, cette bourgeoisie agraire repose sur des bases économiques anticonstitutionnelles. Elle ce compose généralement des propriétaires qui ont plus de 200 ha de terre, propriétés illégales, sauf certaines exceptions. Ces domaines représentent moins de $1 \%$ des exploitations privées agricoles, mais $22 \%$ de la surface cultivable, produisant presque la moitié du revenu agricole du secteur privé. Ces propriétaires proviennent en partie de la bureaucratie nouvelle: anciens fonctionnaires gouvernementaux, intermédiaires administratifs enrichis par le commerce ou la corruption aux dépens des fonds publics. Ce groupe ne peut pas être désigné comme une catégorie exclusivement agraire, car ses intérêts ne se tournent que partiellement vers l'agriculture. Il doit plutôt être considéré comme une fraction de la bourgeoisie bureaucratique et gouvernernentale, qui occupe aujourd'hui des positions dans tous les secteurs de l'économie nationale. Il n'est pas lié directement au sol comme l'est la paysannerie, il considère la terre plutôt comme un simple moyen de production. Il est désigné par le peuple comme les agricultores nylon, car leur sens de la paysannerie est aussi artificiel que leurs propriétés sont illégales. Pourtant, celles-ci sont mécanisées et modernisées au plus haut point et c'est là que se réalisent les progrès les plus considérables de l'agriculture mexicaine. Par là même, ces propriétaires sont intéressés au développement capitaliste de l'agriculture ; mais les bases actuelles de la Réforme agraire au Mexique ne rendent possibles ni ce développement, ni une véritable collectivisation de l'agriculture. Et c'est pour cela que cette couche est liée à une certaine structure politique qui, bien qu'elle ait ses sources dans la Révolution, en est cependant détournée de plus on plus. Ce n'est donc pas ici qu'on trouvera la partie politiquement avancée de la bourgeoisie, mais dans une autre fraction, liée à l'industrie nationale, qui à intérêt au progrès de la Réforme agraire par lequel le marché intérieur se trouverait élargi. La grande bourgeoisie agrarienne est toujours menacée par une nouvelle application de la Réforme agraire. Elle cherche donc à tirer des profits 
toujours plus grands de l'agriculture et à se lier avec des éléments politiques hostiles à cette Réforme.

f) La couche paysanne intermédiaire. - Si l'on retient l'extension de la propriété privée comme caractéristique formelle pour délimiter les fractions sociales composant la population agricole, on remarquera que l'une d'elles n'est pas citée, celle des propriétaires do 5 à 25 ha. En effet, il s'agit ici d'une couche intermédiaire qu'on ne peut assimiler ni aux paysans parcellaires, ni à la bourgeoisie rurale. Ses domaines ne représentent que $3 \%$ des propriétés privées, mais sa participation au revenu du secteur privé de l'agriculture est dix fois plus élevée que celui des petits paysans parcellaires. La plupart du temps, elle a reçu ses titres dé propriété de la Réforme agraire, et c'est en son sein qu'on trouve le paysan indépendant, entreprenant, qui s'est acquis un petit capital en général grâce à l'utilisation judicieuse du bétail de trait et souvent aussi par l'emploi de travailleurs salariés. Étant donné que cette couche ne présente pas les caractères d'une classe originale, elle tend à s'adapter plutôt à la structure économique régionale de la zone dans laquelle se trouvent ses propriétés. Ainsi, dans les régions où prédominent les microfundia et où sévit le chômage déguisé, elle a intérêt à donner une partie de ses terres en métayage, ou à y employer une main-d'oeuvre bon marché. Le capital ainsi accumulé est investi dans le commerce, puisque dans ces régions le manque de terre leur interdit d'étendre leurs propriétés. Elle aura donc tendance à s'assimiler à la bourgeoisie commerçante des agglomérations rurales, Dans les autres régions, moins peuplées, les tendances vers une agriculture mécanisée, capitaliste, pourront se manifester dans des conjonctures favorables. Enfin, il n'est pas exclu qu'elle tombe - ou retombe - dans la classe dos petits paysans, sous le poids de la concurrence de la moyenne et de la grande bourgeoisie agrarienne. En tout cas, ces propriétés de plus de 5 à 25 ha permettent de dépasser le niveau de subsistance, mais difficilement d'atteindre l'accumulation capitaliste. Cette couche se trouve donc, aussi bien par l'extension de ses propriétés que par la nature de son économie, dans une situation intermédiaire ; 
elle est plus ou moins affectée dans son devenir par les relations qui caractérisent les autres groupements de la société agraire.

\section{L'agriculture capitaliste et ses classes.}

$\underline{\text { Retour à la table des matières }}$

Le développement de la production agricole au Mexique, depuis quelques années, s'effectue dans les régions Nord du pays, qui ont été aménagées le plus souvent avec la participation du gouvernement. On y exploite des cultures d'exportation destinées aux États-Unis (coton, et cultures vivrières telles que la tomate, les olives, les raisins, etc.), et aussi au marché national. Ces investissements profitent à la nouvelle bourgeoisie agraire capitaliste. On ne trouve pas dans cette région la vieille structure agraire caractéristique du reste du pays. Ici affluent les paysans sans terre, déracinés de leurs communes, du Centre et du Sud. Ils y arrivent comme journaliers migrants, et parcourent des circuits déterminés, qui les entraînent parfois vers les exploitations agricoles des États-Unis. Sans attaches avec cette terre inconnue et nouvelle, généralement sans leurs familles et sans biens, ces ouvriers agricoles ont aussi brisé leurs liens avec les structures sociales traditionnelles. Ils peuplent les villes nouvelles, et sont intégrés dans une économie monétaire où leur force de travail n'est qu'une marchandise bon marché. Les entreprises agricoles pour lesquelles ils travaillent n'ont rien de commun avec les haciendas; les relations entre patron et ouvrier sont désormais des relations nettement capitalistes. La Révolution avait promis à ces paysans une parcelle de terre propre ; la Réforme agraire leur en a donné l'espoir, et la législation agraire a consacré leur droit à cet égard. Mais pour eux, il n'en est encore rien. Dans ces conditions, la lutte sociale n'est plus seulement une lutte pour la terre : il s'agit de s'assurer, d'abord, d'un salaire, d'un contrat, de prestations sociales. Au lieu des Ligues de Communautés agraires qui caractérisaient l'agitation dans les campagnes pendant la période révolu- 
tionnaire jusqu'à 1940, on trouve, aujourd'hui, les syndicats d'ouvriers agricoles. C'est dans ces conditions que l'économie agricole se développe. Une bourgeoisie agraire capitaliste est un prolétariat agricole en pleine évolution, tel est la base du développement économique de l'agriculture mexicaine, particulièrement dans les régions où la forte croissance démographique est due aux migrations internes. C'est pourquoi nous considérons les relations entre ces deux classes comme étant appelées à jouer un rôle de plus en plus important dans l'avenir du pays. Or, ces relations sont des relations d'opposition, non seulement parce qu'elles représentent un cas du développement capitaliste en pays sous-développé, mais aussi, et plus particulièrement, en vertu de la structure agraire du Mexique que nous avons essayé de décrire dans ces pages.

Mexico. 